\title{
Model building and verification for active control of microvibrations with probabilistic assessment of the effects of uncertainties
}

\author{
G S Aglietti ${ }^{1}$, R S Langley ${ }^{2}$, E Rogers $^{3 *}$ and S B Gabriel ${ }^{1}$ \\ ${ }^{1}$ School of Engineering Sciences, University of Southampton, UK \\ ${ }^{2}$ Department of Engineering, University of Cambridge, UK \\ ${ }^{3}$ School of Electronics and Computer Science, University of Southampton, UK
}

\begin{abstract}
Microvibrations, generally defined as low-amplitude vibrations at frequencies up to $1 \mathrm{kHz}$, are of critical importance in a number of areas. It is now well known that, in general, the suppression of such microvibrations to acceptable levels requires the use of active control techniques which, in turn, require sufficiently accurate and tractable models of the underlying dynamics on which to base controller design and initial performance evaluation. Previous work has developed a systematic procedure for obtaining a finite-dimensional state-space model approximation of the underlying dynamics from the defining equations of motion, which has then been shown to be a suitable basis for robust controller design. In this paper, the experimental validation of this model prior to experimental studies is described in order to determine the effectiveness of the designed controllers. This includes details of the experimental rig and also the use of methods for assessing the safety of the resulting structure against uncertain parameters.
\end{abstract}

Keywords: microvibrations, modelling, active control, uncertainty assessment

$\begin{array}{ll}\text { NOTATION } & \\ d & \text { piezoelectric constant } \\ e & \text { electric field } \\ E & \text { Young's modulus } \\ f & \text { column vector of forces } \\ g & \text { safety margin } \\ h & \text { thickness } \\ \mathbf{M} & \text { inertia matrix } \\ P & \text { probability } \\ q & \text { generalized forces } \\ s & \text { shape function vector } \\ S & \text { shape functions } \\ T & \text { kinetic energy } \\ U & \text { potential energy } \\ v & \text { voltage } \\ \boldsymbol{V} & \text { voltage vector } \\ w & \text { out-of-field displacement } \\ \beta & \\ & \text { safety index }\end{array}$

The MS was received on 8 May 2003 and was accepted after revision for publication on 27 January 2004.

* Corresponding author: School of Electronics and Computer Science, University of Southampton, Highfield, Southampton, Hampshire SO17 1BJ, UK.

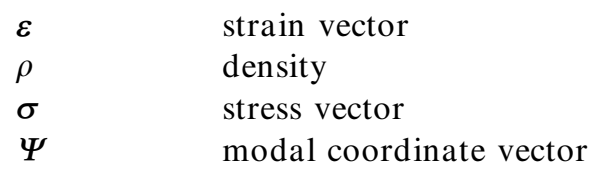

\section{INTRODUCTION}

'Microvibrations' is the term used to describe lowamplitude vibrations that occur at frequencies up to $1 \mathrm{kHz}$ and that have often been neglected in the past due to the low levels of disturbances they induce. In recent years, however, the need to suppress the effects of microvibrations has become much more necessary. This is especially true for spacecraft structures where, due to the ever-increasing requirements to protect sensitive payloads, such as optical instruments or microgravity experiments, there is a pressing need to obtain a very high level of microvibration-induced vibration suppression (see, for example, reference [1] for further background information). In effect, such vibrations on board spacecraft are produced by the functioning of onboard equipment such as reaction wheels, gyroscopes, thrusters, electric motors, etc., which propagate 
through the satellite structure towards sensitive equipment (receivers), thereby jeopardizing their correct functioning.

From a practical standpoint, the reduction of the vibration level at a sensitive location of a structure can be attempted by action at the source(s), receiver(s) and along the vibration path(s). At the source(s), this action consists of attempting to minimize the amplitude(s) of the vibration(s) by, for example, placing equipment on appropriate mountings. The same approach is commonly attempted at the receiver(s) but with the basic objective of sensitivity reduction. Finally, along the vibration path(s), modifications of structural elements or re-location of equipment is attempted with the aim of reducing the mechanical coupling(s) between source(s) and receiver(s).

All of the approaches described above can be based on so-called passive damping technology and, for routine applications, an appropriate combination of them is often capable of producing the desired levels of dynamic disturbance rejection. The use of active control techniques in such cases would only be as a last resort to achieve the desired performance.

The requirements of the new generation of satellitebased instruments are such that only active control can be expected to provide the required levels of microvibration suppression. To investigate the use of active control to suppress such vibrations in a structure, computationally feasible models that retain the core features of the underlying dynamics are clearly required. The most obvious approach to the development of such models is to use finite element methods (FEMs) (see, for example, reference [2]) due to the accuracy available with a sufficiently fine mesh. The only difficulties with this approach are the computational intensity of the models and the fact that they are not in a form directly compatible with feedback control systems design. They can, however, be used, as in this work, to verify that the modelling strategy employed produces 'realistic' models on which to base controller design and evaluation.

Alternatives to FEMs can be classified as elastic wave methods, variational methods and mechanical impedance based methods respectively. A detailed study of the advantages and disadvantages of these methods, together with background references on each of them, can be found in references [3] and [4]. Based on this study, a Lagrange-Rayleigh-Ritz (LRR) method has been developed (again see references [3] and [4]) to produce state-space models on which to base controller design.

In this paper, the use of this modelling technique in controller design and initial evaluation is first illustrated. The focus is on the design of controllers based on linear quadratic optimal control theory for massloaded panels, which are widely recognized as an acceptable compromise between problem complexity and the need to gain useful insights into the benefits (and limitations) of active control schemes in this general area. Of course, the model is not a complete representation of the system dynamics and prior to actual implementation studies it is essential to compare the actual quality of the model predictions against measured data; clearly the open-loop case needs to be studied first.

This last requirement forms a major part of this paper, where the design of an experimental rig to accurately represent a mass-loaded actively controlled panel is detailed. Comparison of the predicted and measured results then enable the sources of any significant errors to be determined. Also it is shown that this modelling approach enables probabilistic-based studies of the effects of uncertainties in the system's properties to be undertaken. This yields estimates of the probability of an unstable plant and statistical measures of expected performance. In the next section a summary gives the necessary background on mathematical modelling.

\section{SYSTEM DESCRIPTION AND MODELLING}

\subsection{Mass-loaded panel}

The mass-loaded panel arrangement considered here is shown schematically in Fig. 1, where the equipment mounted on the panel is modelled as lumped masses and the disturbances as point forces. The sensors and actuators employed are twin patches of piezoelectric material bonded on to opposite faces of the panel. The bending vibrations of the panel produce stretching and shrinking of the patches depending on whether they are on the top or the bottom of it (see Fig. 2a). Due to the piezoelectric effect, these deformations induce an electric field perpendicular to the panel, which is detected by the electrodes of the patches. The outer electrodes of the patches are electrically connected together and the panel, which is grounded, is used as the other electrode for both patches of the pair when acting as a sensor. The same configuration is used for an actuator, but in this case the electric field is applied externally to produce contraction or expansion of the patch, which then induces a curvature of the panel.

The LRR based procedure used to model this system is based on Lagrange's equations of motion, which in the general case take the form

$$
\frac{\mathrm{d}}{\mathrm{d} t}\left(\frac{\partial T}{\partial \dot{q}_{i}}\right)-\frac{\partial T}{\partial q_{i}}+\frac{\partial U}{\partial q_{i}}=Q_{i}
$$

Here $T$ and $U$ are the kinetic and potential energies of the system and $q_{i}$ and $Q_{i}$ are the $i$ th generalized coordinate and force respectively. For the particular case considered here, the kinetic and potential energies 


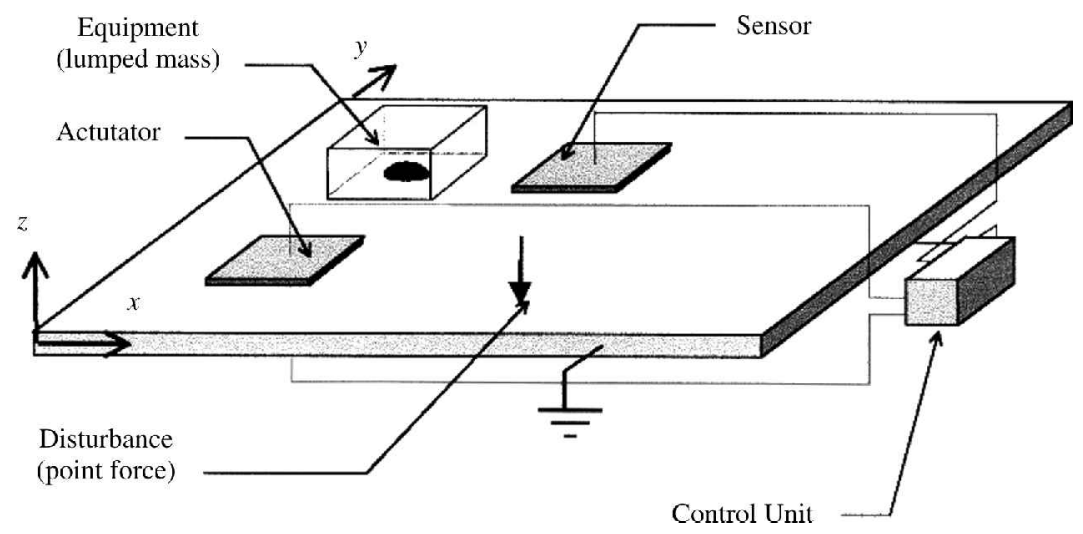

Fig. 1 Mass-loaded panel

(elastic and electric) can be expressed as

$$
T=T_{\mathrm{pl}}+T_{\mathrm{lm}}+T_{\mathrm{pz}}, \quad U=U_{\mathrm{pl}}+U_{\mathrm{pz}}
$$

where $T_{\mathrm{pl}}, T_{\mathrm{lm}}$ and $T_{\mathrm{pz}}$ denote the kinetic energies of the panel, lumped masses and piezoelectric patches respectively. The terms $U_{\mathrm{pl}}$ and $U_{\mathrm{pz}}$ denote the potential energies stored in the panel and the piezoelectric patches respectively.

The displacement field (out-of-plane displacement $w$ ) is described by a superposition of shape functions $S_{m, n}$ (consisting of the first $N=N_{m} \times N_{n}$ modes of the bare panel) multiplied by the time-dependent modal coordinates $\psi_{m, n}$, i.e.

$$
w(x, y, t)=\sum_{m=1}^{N_{m}} \sum_{n=1}^{N_{n}} S_{m, n}(x, y) \psi_{m, n}(t)=\boldsymbol{s}^{\mathrm{T}} \boldsymbol{\Psi}
$$

where the $N \times 1$ column vectors $\boldsymbol{s}$ and $\Psi$ contain the shape functions and modal coordinates respectively. In this work, a simply supported boundary condition is considered and therefore the mode shapes are simply sine functions.

As explained below, the full set of generalized coordinates $q_{i}$, which appears in equation (1), consists of $\Psi$ together with the voltages at the piezoelectric patches. The external excitation consists of $N_{f}$ point forces $F_{j}$ acting on the plate at arbitrary locations. Hence the generalized forces are of the form

$$
Q_{i}=\sum_{j=1}^{N_{f}} F_{j} \frac{\partial w_{j}}{\partial \psi_{i}}
$$

or

$$
Q=\mathbf{S}_{\mathrm{f}} \boldsymbol{f}
$$

where $f$ is the $N_{f} \times 1$ column vector of forces and $\mathbf{S}_{\mathrm{f}}$ is a compatibly dimensioned matrix whose columns are given by the model shape vector $s$ evaluated at the corresponding force locations.

It is now necessary to compute each of the terms in equation (2), starting with the kinetic energies. Each of these terms can be calculated using the standard formula

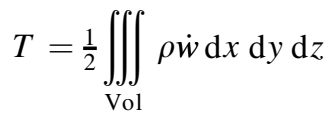

where $\rho$ denotes the material density and Vol denotes the volume. Here it is only necessary to give the final forms, with full details being found in reference [3]. In the case of the transversely vibrating panel, application of equation (6) yields
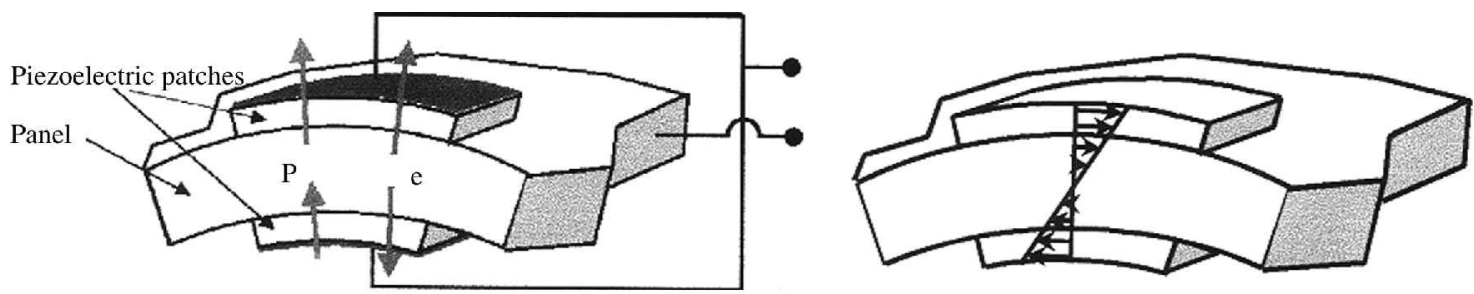

(a)

(b)

Fig. 2 (a) Cross-section of a piezoelectric patch bonded on the panel during deformation (P, poling direction of the piezoelectric material; e, induced electric field). (b) Strain distribution through a piezoelectric patch/panel section 


$$
T_{\mathrm{pl}}=\frac{1}{2} \dot{\boldsymbol{\Psi}}^{\mathrm{T}} \mathbf{M}_{\mathrm{pl}} \dot{\boldsymbol{\Psi}}, \quad T_{\mathrm{pz}}=\frac{1}{2} \dot{\boldsymbol{\Psi}}^{\mathrm{T}} \mathbf{M}_{\mathrm{pz}} \dot{\boldsymbol{\Psi}}
$$

Here $\mathbf{M}_{\mathrm{pl}}$ is the (diagonal) inertia matrix of the panel and the inertia matrix of the patches $\mathbf{M}_{\mathrm{pz}}$ is fully populated, where the off-diagonal entries denote the couplings between the modal coordinates.

Suppose now that there are $N_{1}$ lumped masses on the panel and let $\boldsymbol{s}_{\mathrm{Im}_{i}}, 1 \leqslant i \leqslant N_{1}$, denote the shape vector function at lumped mass $i$. Then the total kinetic energy associated with the lumped masses is given by

$$
T_{\mathrm{lm}}=\frac{1}{2} \dot{\Psi}^{\mathrm{T}} \mathbf{M}_{\mathrm{lm}} \dot{\boldsymbol{\Psi}}, \quad \mathbf{M}_{\mathrm{lm}}=\sum_{i=1}^{N_{1}} \mathbf{M}_{\operatorname{lm}_{i}} \boldsymbol{s}_{\operatorname{lm}_{i}} \boldsymbol{s}_{\operatorname{lm}_{i}}^{\mathrm{T}}
$$

The potential energy of the system is stored as the elastic energy of the panel and the elastic/electric energy of the piezoelectric patches. The elastic energies are directly calculated from the expression

$$
U=\frac{1}{2} \iiint_{\text {Vol }} \varepsilon^{\mathrm{T}} \sigma \mathrm{d} x \mathrm{~d} y \mathrm{~d} z
$$

where $\sigma$ and $\varepsilon$ are the stress and strain vectors respectively. Also by assuming a plane stress condition (see reference [4]) for the panel,

$$
U_{\mathrm{pl}}=\frac{1}{2} \Psi^{\mathrm{T}} \mathbf{K}_{\mathrm{pl}} \Psi
$$

where $\mathbf{K}_{\mathrm{pl}}$ is the panel stiffness matrix. For the piezoelectric patches, the potential energy can be written as the sum of three energy components, i.e.

$$
U_{\mathrm{pz}}=U_{\mathrm{pz}_{\text {elast }}}+U_{\mathrm{pz}_{\text {elastelect }}}+U_{\mathrm{pz}_{\text {elect }}}
$$

where $U_{\mathrm{pz}_{\text {elast }}}$ is the energy stored due to the elasticity of the material, $U_{\mathrm{pz}_{\text {elastelect }}}$ represents the additional energy due to the voltage-driven piezoelectric effect and $U_{\text {pzelect }}$ is the electric energy stored due to the dielectric characteristics of the piezoelectric material employed. To compute the elastic energy in this case, an appropriate model for the stress-strain pattern in the piezoelectric patches must be selected, and here the following assumptions are made in this respect:

1. The electrodes attached to the piezoelectric patches have negligible mass and stiffness.

2. The thickness of the layer of adhesive that connects each of the patches to the panel is negligible compared to that of the patches and is able to transfer all of the shear strain.

3. The natural boundary conditions at the edges of each patch (i.e. $\sigma=0$ ) are not enforced and a compatible strain distribution, as illustrated in Fig. 2b, is assumed through the whole patch.

This last assumption is particularly appropriate if, as here, the patches employed are 'very thin' and 'relatively wide'. Given these assumptions, the same procedure as employed for the panel can be used to write

$$
U_{\mathrm{pz}_{\text {elast }}}=\frac{1}{2} \Psi^{\mathrm{T}} \mathbf{K}_{\mathrm{pz}_{\text {elast }}} \Psi
$$

where the stiffness matrix $\mathbf{K}_{\mathrm{pz}_{\text {clast }}}$ is fully populated.

Suppose now that a patch to be used has a constant thickness $h_{\mathrm{pz}}$ that is thin enough to prevent fringe effects and has a voltage $v$ applied at its electrodes. Then a constant electric field $e=v / h_{\mathrm{pz}}$ can be assumed across the patch and the further stress due to the applied voltages is given by

$$
\sigma_{\text {elect }}=\left(\begin{array}{l}
\sigma_{x_{\text {elect }}} \\
\sigma_{y_{\text {elect }}}
\end{array}\right)=\frac{E_{\mathrm{pz}}}{1-v^{2}}\left(\begin{array}{c}
d_{x z}+v d_{y z} \\
d_{y z}+v d_{x z}
\end{array}\right) e
$$

Here $d_{x z}$ and $d_{y z}$ are the piezoelectric constants of the material, which is assumed to have a polling direction $z$ perpendicular to the plate. Hence $U_{\mathrm{pz}_{\mathrm{elastelect}}}$ can be calculated as

$$
U_{\mathrm{pz}_{\text {elastelect }}}=\frac{1}{2} \iiint_{\mathrm{pz}} \sigma_{\text {elect }}^{\mathrm{T}} \varepsilon \mathrm{d} x \mathrm{~d} y \mathrm{~d} z
$$

In the case of $N_{p}$ patches, the electric field in patch $i$ can be written in the form $e_{i}(t)=\boldsymbol{V}(t)^{\mathrm{T}} \boldsymbol{p}_{i}$, where $\boldsymbol{V}(t)$ is the $N_{p} \times 1$ vector whose entries are the patch voltages $\left(v_{i}\right)$ and the $N_{p} \times 1$ vector $\boldsymbol{p}_{i}$ has zero entries except for entry $i$, which is equal to $1 / h_{\mathrm{pz}_{i}}$. Also, by assuming that $d_{x z}=d_{y z}=d_{z}$ and substituting the assumed form of $\sigma_{\text {elect }}$ in equation (13) into equation (14), it is possible to write the elastoelectric energy stored in the $N_{p}$ patches as

$$
U_{\mathrm{pz}_{\text {elastelect }}}=V^{\mathrm{T}} \mathbf{K}_{\mathrm{pz}_{\text {elastelect }}} \Psi
$$

The electrical energy stored in the piezoelectric material can be expressed as

$$
U_{\mathrm{pz}_{\text {elect }}}=\frac{1}{2} \iiint_{\mathrm{pz}} e d \mathrm{~d} x \mathrm{~d} y \mathrm{~d} z
$$

where $e$ is the electric field and $d$ is the electric displacement (charge/area). For each patch, the electric displacement is

$$
d_{i}=\varepsilon_{\mathrm{pz}_{i}} \boldsymbol{p}_{i}^{\mathrm{T}} v_{i}
$$

where $\varepsilon_{\mathrm{pz}_{i}}$ is the dielectric constant of the piezoelectric material which forms the $i$ th patch. Hence an equivalent expression for the stored electric energy is

$$
U_{\mathrm{pz}_{\text {elect }}}=\frac{1}{2} \boldsymbol{V}^{\mathrm{T}} \mathbf{K}_{\mathrm{pz}_{\text {elect }}} \boldsymbol{V}
$$

where the elements of the matrix $\mathbf{K}_{\mathrm{pz}_{\text {elect }}}$ are the capacitances of the piezoelectric patches.

At this stage, all of the energy terms are available as functions of the generalized coordinates, i.e. the modal coordinates and the voltages at the patches written in column vector form as $\Psi$ and $\boldsymbol{V}$ respectively. Hence straightforward application of Lagrange's equations of motion (1) yields the second-order matrix differential 
equation model

$$
\begin{aligned}
& \left(\mathbf{M}_{\mathrm{p} 1}+\mathbf{M}_{\mathrm{pz}}+\mathbf{M}_{\mathrm{lm}}\right) \ddot{\boldsymbol{\Psi}}+\left(\mathbf{K}_{\mathrm{pl}}+\mathbf{K}_{\mathrm{pz}_{\text {elast }}}\right) \boldsymbol{\Psi}+\mathbf{K}_{\mathrm{pz}_{\text {elastelect }}^{\mathrm{T}}} \boldsymbol{V} \\
& \quad=Q \\
& \mathbf{K}_{\mathrm{pz}_{\text {elastelect }}} \boldsymbol{\Psi}+\mathbf{K}_{\mathrm{pz}_{\text {elect }}} \boldsymbol{V}=0
\end{aligned}
$$

The first equation in (19) results from first differentiating the energy terms with respect to the modal coordinates and writing the results in terms of the column vectors $\Psi$ and $V$ and the second from an identical set of operations but with differentiation with respect to the patch voltages. These operations assume that all modal coordinates and voltages are degrees of freedom (DOFs) of the system.

In the case when all patches act as actuators, their voltages are externally driven and hence the second equation in (19) is redundant. If all patches are to be used as sensors, the second equation in (19) can be used to obtain an expression for the voltages as a function of the modal coordinates. This expression can then be substituted into the first equation in (19) to give a complete set of equations in the unknown modal coordinates.

The most general case arises when some of the patches act as actuators and others as sensors. In such a case it is necessary to partition the matrix $\mathbf{K}_{\mathrm{pz}_{\text {elastelect }}}$ to separate out actuator and sensor contributions. To do this, let $\boldsymbol{v}_{\mathrm{a}}$ and $\boldsymbol{v}_{\mathrm{s}}$ be the subvectors of the voltages at the actuators and sensors respectively, and partition $\mathbf{K}_{\mathrm{pz}_{\text {clastelect }}}$ conformally as $\mathbf{K}_{\mathrm{pz}_{\text {elastelect }}}=\left[\mathbf{K}_{\mathrm{pza}_{\text {elastelect }}}, \mathbf{K}_{\mathrm{pzs}_{\text {elasselect }}}\right]$. Then the first equation in (19) can be rewritten as

$$
\begin{gathered}
\mathbf{M} \ddot{\boldsymbol{\Psi}}+\mathbf{C}_{\mathrm{s}} \dot{\boldsymbol{\Psi}}+\left(\mathbf{K}_{\text {elast }}+\mathbf{K}_{\mathrm{pzs}}\right) \boldsymbol{\Psi} \\
=-\mathbf{K}_{\text {pza }_{\text {elastelect }}^{\mathrm{T}}} \boldsymbol{v}_{\mathrm{a}}+\boldsymbol{s}_{\mathrm{f}}^{\mathrm{T}} \boldsymbol{f}
\end{gathered}
$$

where all inertia elements are included in the matrix $\mathbf{M}$ and all stiffness elements in the matrix $\mathbf{K}_{\text {elast }}$. Also,

$$
\mathbf{K}_{\mathrm{pzs}}=-\mathbf{K}_{\mathrm{pzs} \text { elastelect }}^{\mathrm{T}} \mathbf{K}_{\mathrm{pzs}_{\text {elect }}}^{-1} \mathbf{K}_{\mathrm{pzs}_{\text {elastelect }}}
$$

represents the contribution to the stiffness from the piezoelectric energy stored in the patches acting as sensors, where $\mathbf{K}_{\text {pzs }_{\text {elect }}}$ is the submatrix of $\mathbf{K}_{\mathrm{pz}_{\text {elect }}}$ corresponding to the sensors. In addition, structural damping has been added to the system by including the term $\mathbf{C}_{\mathrm{s}} \dot{\boldsymbol{\Psi}}$.

All the necessary matrices in this model can be computed directly given the relevant data (dimensions, material properties, patch positions, etc.). Also, the model of equation (20) can easily be written in statespace form as

$$
\begin{aligned}
& \dot{\boldsymbol{x}}=\mathbf{A} \boldsymbol{x}+\mathbf{B}_{v} \boldsymbol{v}_{\mathrm{a}}+\mathbf{B}_{\mathrm{f}} f \\
& \boldsymbol{v}_{\mathrm{s}}=\mathbf{C}_{v} \boldsymbol{x} \\
& w_{\text {out }}=\mathbf{C}_{\mathrm{w}} \boldsymbol{x}
\end{aligned}
$$

where

$$
\begin{aligned}
& \boldsymbol{x}=\left[\begin{array}{c}
\boldsymbol{\Psi} \\
\dot{\boldsymbol{\Psi}}
\end{array}\right], \quad \mathbf{A}=\left[\begin{array}{cc}
0 & \boldsymbol{I} \\
-\mathbf{M}^{-1} \mathbf{K} & -\mathbf{M}^{-1} \mathbf{C}_{\mathrm{s}}
\end{array}\right] \\
& \mathbf{B}_{\mathrm{v}}=\left[\begin{array}{c}
0 \\
\mathbf{M}^{-1} \mathbf{K}_{\text {pza }_{\text {elastelect }}^{\mathrm{T}}}^{\mathrm{T}}
\end{array}\right], \quad \mathbf{B}_{\mathrm{f}}=\left[\begin{array}{c}
0 \\
\mathbf{M}^{-1} \boldsymbol{s}_{\mathrm{f}}^{\mathrm{T}}
\end{array}\right] \\
& \mathbf{C}_{\mathrm{v}}=\left[\begin{array}{lll}
-\mathbf{K}_{\text {pzs elect }}^{-1} & \mathbf{K}_{\text {pzs } \text { elastelect }} & 0
\end{array}\right], \quad \mathbf{C}_{w}=\left[\begin{array}{ll}
\boldsymbol{s}_{\text {out }}^{\mathrm{T}} & 0
\end{array}\right]
\end{aligned}
$$

Here $w_{\text {out }}$ is the output displacement at the particular locations specified by the mode shape vectors $\boldsymbol{s}_{\text {out }}$ and $\mathbf{K}$ denotes the total stiffness matrix defined from equation (21) $\left(\mathbf{K}=\mathbf{K}_{\text {elast }}+\mathbf{K}_{\text {pzs }}\right)$.

Using this state-space description, it is possible to begin in-depth investigations of the potential (or otherwise) of active control schemes in this general area. To be practically relevant, however, it is clearly essential that the model to be used for controller design adequately represents the underlying plant dynamics. In the next section of this paper the development of an experimental rig for this purpose is described.

\section{EXPERIMENTAL RIG AND TESTING}

A test rig for the simply supported panel (see Table 1 for all details subsequently used in this paper) configuration described in the previous section has been built in order to verify the results produced by the mathematical models. One of the main problem areas was the realization of the simple supports along the edge of the panel. This problem, also addressed in detail by Aglietti and Cunningham [5], was solved by suspending the panel horizontally using shims. This set-up produces a negligible rotational stiffness along the edge of the panel due to the high bending flexibility of the shim. At the same time, the high in-plane stiffness of the shim, which is clamped along its upper and lower edges,

Table 1 Dimensions and material properties of the simply supported panel

\begin{tabular}{lll}
\hline Panel & $a=304.3 \mathrm{~mm}$ & $E=71 \times 10^{9} \mathrm{~Pa}$ \\
& $b=203.2 \mathrm{~mm}$ & $\rho=2705 \mathrm{~kg} / \mathrm{m}^{3}$ \\
& $h=1.453 \mathrm{~mm}$ & $v=0.33$ \\
Lumped mass & $x=50.8 \mathrm{~mm}$ & $m=38 \mathrm{~g}$ \\
Actuator & $y=152.4 \mathrm{~mm}$ & \\
& $x_{\mathrm{a}_{1}}=50.8 \mathrm{~mm}$ & $E=63 \times 10^{9} \mathrm{~Pa}$ \\
& $x_{\mathrm{a}_{2}}=101.6 \mathrm{~mm}$ & $\rho=7650 \mathrm{~kg} / \mathrm{m}^{3}$ \\
& $y_{\mathrm{a}_{1}}=25.4 \mathrm{~mm}$ & $v=0.3$ \\
& $y_{\mathrm{a}_{2}}=76.2 \mathrm{~mm}$ & $d=1.66 \times 10^{-10} \mathrm{~m} / \mathrm{V}$ \\
& $h_{\mathrm{pz}}=0.19 \mathrm{~mm}$ & $\varepsilon=1700 \varepsilon^{\circ}$ \\
& $x_{\mathrm{S}_{1}}=76.2 \mathrm{~mm}$ & $E=63 \times 10^{9} \mathrm{~Pa}$ \\
& $x_{\mathrm{S}_{2}}=127 \mathrm{~mm}$ & $\rho=7650 \mathrm{~kg} / \mathrm{m}^{3}$ \\
$y_{\mathrm{S}_{1}}=101.6 \mathrm{~mm}$ & $v=0.3$ \\
$y_{\mathrm{S}_{2}}=152.4 \mathrm{~mm}$ & $d=1.66 \times 10^{-10} \mathrm{~m} / \mathrm{V}$ \\
& $h_{\mathrm{pz}}=0.19 \mathrm{~mm}$ & $\varepsilon=1700 \varepsilon^{\circ}$ \\
& &
\end{tabular}




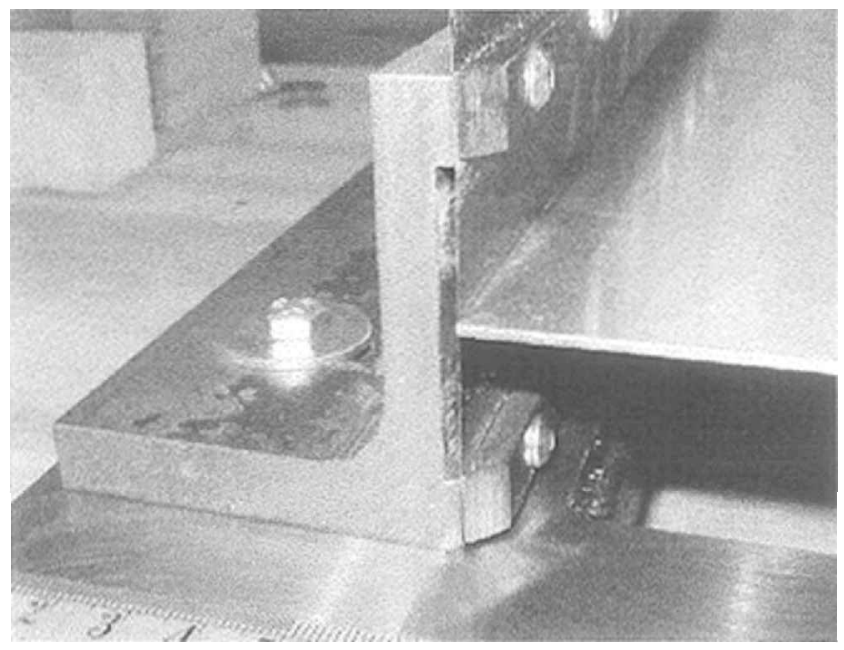

Fig. 3 Detail of the panel support

restrains the out-of-plane (vertical) movements of the edge of the panel.

Figure 3 shows the practical implementation of this support, and more details can be found in Aglietti and Cunningham [5]. The corner pieces (L-cross-section segments of steel beam) on to which the shims are constrained are then bolted to a rigid frame. Another advantage of this type of support is that the out-of-plane flexibility of the shims allows expansion or contraction of the panel (due to temperature changes) without inducing large in-plane pre-stresses in the panel.

The corner pieces (L-sections) that support the shims were bolted on to a frame composed of a steel plate, with a rectangular cut-out machined in the centre of the plate to the dimensions of the aluminium alloy panel and four steel $\mathrm{U}$-section beams welded under the plate to increase its stiffness The cut-out in the plate is necessary in order to avoid standing acoustic waves that otherwise would be produced in the gap (cavity) between the aluminium panel and the steel plate. The four L-section steel beams were machined with a channel to produce a gap behind the shims.

The piezoelectric patches were bonded to the panel using epoxy. The complete test rig was suspended in a frame using four tension springs to provide freely supported boundary conditions to the supporting structure. The reason for having the frame 'floating' was that any attempt to constrain the frame to the ground resulted in flexible modes of the frame being introduced in the frequency range under investigation. This was arbitrarily chosen as $1-500 \mathrm{~Hz}$ to include the first five modes of the panel. Finally, the lumped mass was bonded to the panel. This mass consists of two small solid steel cylinders bonded on to the opposite faces of the panel at the same location. This arrangement was used in order to maintain symmetry with respect to the panel middle surface and to keep the centre of mass of the lumped mass on the middle surface of the panel so that the moments of inertia of the lumped mass arrangement were minimized. Figure 4 shows the experimental arrangement.

\subsection{FE model}

\subsubsection{Panel with piezoelectric patches}

A finite element (FE) model (Fig. 5) of the assembly has been constructed using the commercial software package ANSYS. The model was composed of layered shell elements (Shell91), with the areas of the piezoelectric

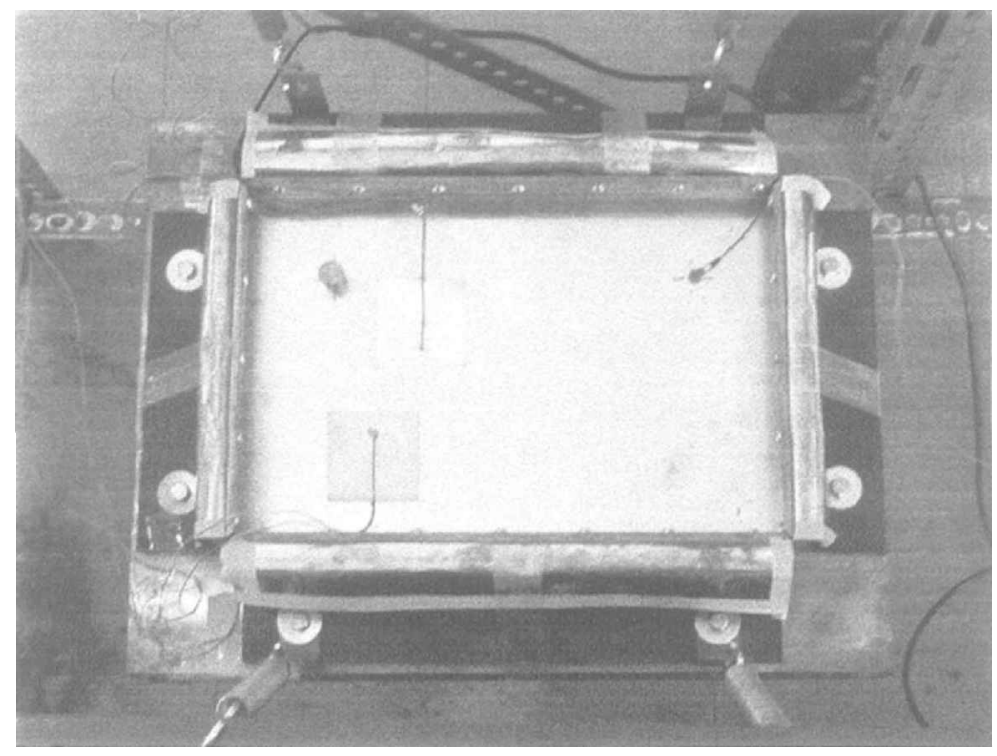

Fig. 4 Piezoelectric actuated mass-loaded panel 


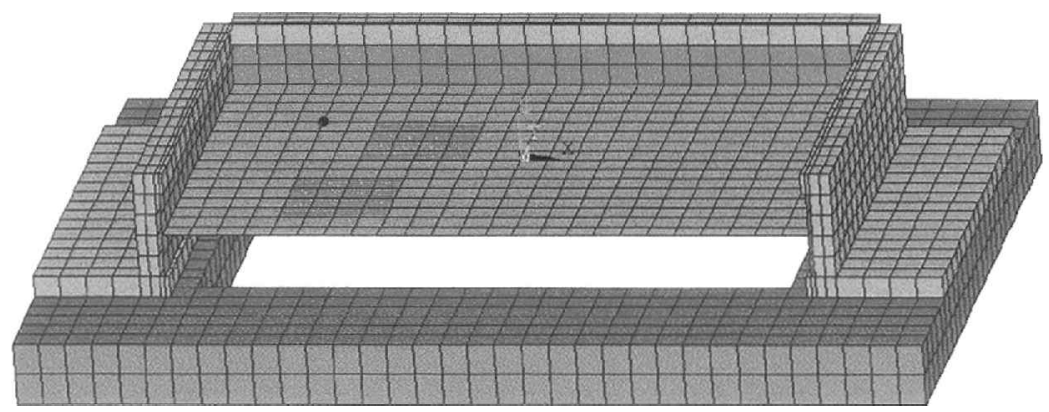

Fig. 5 FE model of the experimental rig (the front L-section beam is removed for clarity)

patches modelled using three layers (piezoelectric material upper patch/aluminium/piezoelectric material lower patch). For the rest of the panel a single layer of aluminium was used. In the FE model, the driving force produced by the contraction/expansion of the piezoelectric patches used as actuators, when the input voltage is applied, was produced by applying moments along the edges of the piezoelectric patch.

This was done in order to keep the FE model as simple as possible without adding further degrees of freedom (DOFs) such as voltages or temperatures to produce shrinking/expansion of the patches. The moment applied to the edge of the patch is based on that originally developed and tested for beams by Brennan et al. [6].

\subsection{Complete rig}

In the FE model of the whole assembly (i.e. the panel and supporting structure), the L-section beams that support the shims and the metal strips clamping the shims have been modelled using solid elements while the supporting plate and the U-channels welded underneath were modelled using shell elements. The L-sections were coupled to the frame by merging the nodes in the areas where the connecting bolts between these elements were located. The modes of vibration and the associated natural frequencies were first calculated with the whole assembly freely supported.

\section{TESTS AND DISCUSS ION OF RESULTS}

Before testing, the size and material properties of the aluminium panel were experimentally verified in order to confirm the input data to be used in the mathematical models. Using the experimental set-up described previously, the four transfer functions characterizing this plant (i.e. input actuator signal-output sensor signal, input actuator signal-output displacement on the panel, input disturbance force-output sensor signal, input disturbance force-output displacement on the panel) were retrieved experimentally.
To reproduce the input disturbance force, the panel was tapped using an Endevco impact hammer with an Isotron force transducer. The panel was tapped at the location $x=254 \mathrm{~mm}$ and $y=51 \mathrm{~mm}$. The response of the panel was retrieved using a miniature accelerometer at $x=254 \mathrm{~mm}$ and $y=152 \mathrm{~mm}$. The weight of the accelerometer was $0.6 \mathrm{~g}$ and therefore its impact on the dynamics of the panel was neglected. The voltage produced at the electrodes of the piezoelectric patch was acquired using a Signal Processing Limited fourchannel data acquisition suite connected to a personal computer which operated using Matlab software.

As an example, Fig. 6 shows a comparison between the experimentally measured transfer function input actuator signal-output sensor signal and the results obtained using the LRR model. The comparison between experimental data and the theoretical predictions of the LRR model has confirmed the quality of the LRR modelling technique. The data here are limited to between 50 and $500 \mathrm{~Hz}$, the reason being that beyond approximately $500 \mathrm{~Hz}$ the frame used to support the panel in the experiments starts to deform and has

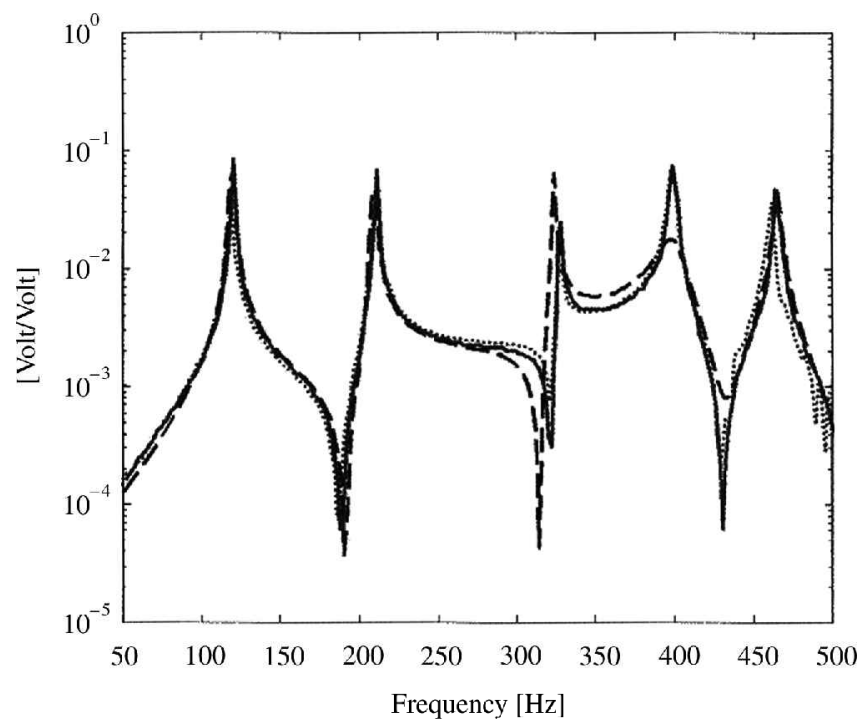

Fig. 6 Transfer function between the input at the actuator and sensor output (dotted line, experimental results; dashed line, FEM; continuous line, LRRM) 
resonances that interfere with the panel dynamics. Similarly, at low frequency (say a few $\mathrm{Hz}$ ) the panel plus frame (supported by soft springs) exhibit rigidbody modes, which are not included in the theoretical models.

Also, the FE model produced excellent results, but it must be noted that the FE model had approximately 3500 DOFs while the LRR model only considers the first 6 by 6 mode shapes (36 DOFs). In addition, the good comparison between $\mathrm{FE}$ model results and experimental data confirms that the method used to simulate the effect of the patches with line moments along the edges is accurate. The voltage at the electrodes of the patch in the FE model is obtained from the average strain in the patch multiplied by the piezoelectric constant.

\section{CONTROLLER DESIGN}

Given the state-space model of equations (22) to (24), it is possible to design a controller whose objective is to minimize the displacement at a specified point on the panel in the presence of point force disturbances acting at other location(s). This can be undertaken by any of the currently available controller design tools. Here (for illustrative purposes only) linear quadratic optimal control is used, where the aim of the controller is the minimization of the performance index:

$$
J=\frac{1}{2} \int_{0}^{\infty}\left(w_{\text {out }}^{\mathrm{T}} \mathbf{Q} w_{\text {out }}+v_{\mathrm{a}}^{\mathrm{T}} \mathbf{R} v_{\mathrm{a}}\right) \mathrm{d} t
$$

where $\mathbf{Q}$ (positive semi-definite) and $\mathbf{R}$ (positive definite) are weighting matrices of compatible dimensions to be selected by the designer. Appropriate selection of the entries in the weighting matrices $\mathbf{Q}$ and $\mathbf{R}$ allows a balance between the minimization of the output of the plant $w_{\text {out }}$ (which is our main objective) and the minimization of the control effort. A sample design is shown in Fig. 7 in the form of frequency response data.

\section{UNCERTAINTY ANALYSIS}

In structural and mechanical design it is clearly essential to allow for the fact that uncertainties can exist in the properties of the designed system and in the applied loading. The traditional means of dealing with this has been through the use of factors of safety, which are developed and refined on the basis of experience and historical evidence. For systems where efficient design is of the utmost importance [and this is clearly the case in the (eventual) applications area considered here], it could easily be the case that the traditional safety factors may be too conservative and hence optimal efficiency cannot be achieved.

The most direct approach to a theoretical reliability assessment is to attempt to derive the statistics of the system response (and the failure probability) from a knowledge of the statistical properties of the system and the applied loads. In the case of complex systems, however, two major problems can arise with this approach. These are that the evaluation of the response statistics can present severe mathematical and numerical difficulties and also the statistical properties of the

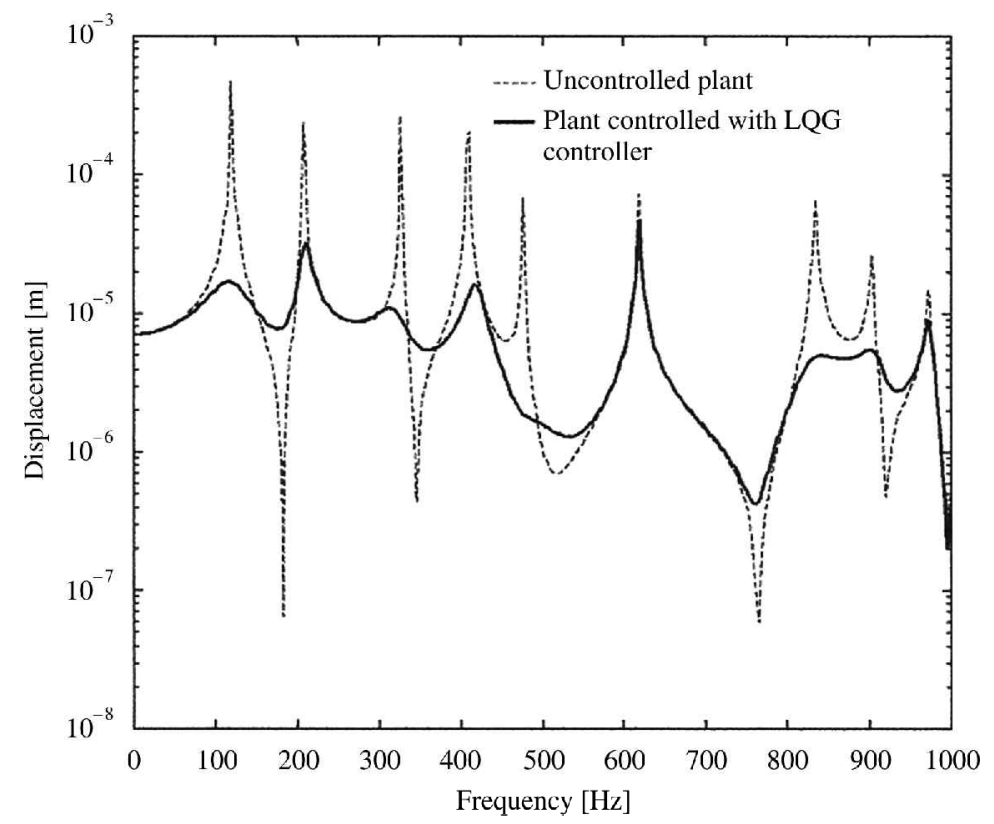

Fig. 7 Frequency response to a $1 \mathrm{~N}$ input disturbance force for nominal plant with and without active control 
system and loading may not be known in any detail. This has led to the development of a number of approximate analytical methods and, in particular, asymptotic reliability analysis, FORM (first-order reliability method) and SORM (second-order reliability method) (see, for example, reference [7]), and commercial software codes are now available for application of the latter two methods to FE models [8].

The key feature of FORM is that it linearizes the limit state surface about the 'design point'. This point is chosen to make this approximation as accurate as possible. If the limit state surface is actually linear then the analysis is trivial and a closed solution for the reliability can be derived. For the non-linear case, FORM is an approximate numerical method specifically developed to deal with non-linear failure surfaces.

In this paper, as an example, the use of FORM is used for the particular structures considered. The numerical results given here demonstrate that the FORM probability agrees with the Monte Carlo result and this approach gives a good approximation for the class of structures. Next the relevant background results are given (with full details in, for example, reference [9] and the relevant cited references). Note also that alternatives to the FORM method exist. These include the normal tail approximation $[\mathbf{1 0}]$ and the weighted fractile approximation [11]. A clear area of further work would obviously be to compare these approaches against the results obtained here by the FORM method.

If a number of properties of an engineering structure are taken to be random, then the response to applied loading (of either deterministic or random magnitude) will also be random. Here the random variables that describe the structure and the applied loading are taken to form a vector $\boldsymbol{x}$ of dimension $n$, and hence the statistics of the system are fully described by the joint probability density function $\rho(\boldsymbol{x})$. For a given $\boldsymbol{x}$, the structure will either fail under the applied loading or exhibit safe behaviour. Hence the condition of the structure can be described by a safety margin $g(\boldsymbol{x})$ so that the structure has failed if $g(x) \leqslant 0$ and is safe if $g(\boldsymbol{x})>0$. It then follows that the probability of failure can be written in the form

$$
P_{\mathrm{F}}=\int_{g(\boldsymbol{x}) \leqslant 0} \rho(\boldsymbol{x}) \mathrm{d} x
$$

To proceed, it is convenient to express $\rho(\boldsymbol{x})$ in the form

$$
\rho(\boldsymbol{x})=\mathrm{e}^{-f(\boldsymbol{x})}
$$

where $f(\boldsymbol{x})=-l(\boldsymbol{x})$ and $l(\boldsymbol{x})$ is termed the log likelihood function. Also it can be shown [9] that $P_{\mathrm{F}}$ can be approximated by

$$
P_{\mathrm{F}} \approx(2 \pi)^{N / 2}|X|^{-1 / 2} \rho(\hat{\boldsymbol{x}}) \mathrm{e}^{\beta^{2} / 2} \Phi(-\beta)
$$

where

$$
\begin{aligned}
& X_{i j}=\frac{\partial^{2} f}{\partial x_{i} \partial x_{j}}+\left(\frac{|\nabla f|}{|\nabla g|}\right) \frac{\partial^{2} g}{\partial x_{i} \partial x_{j}} \\
& \beta=\left(\frac{|\nabla f|}{|\nabla g|}\right) \sigma
\end{aligned}
$$

with

$$
\sigma^{2}=\sum_{i=1}^{N} \sum_{j=1}^{N}\left(X^{-1}\right)_{i j} \frac{\partial g}{\partial x_{i}} \frac{\partial g}{\partial x_{j}}
$$

and all the terms in equations (28) to (30) are to be evaluated at $\hat{\boldsymbol{x}}$, which is defined such that

$$
\min \{f(\boldsymbol{x}): g(\boldsymbol{x})=0\}=f(\hat{\boldsymbol{x}})
$$

This last equation states that $\hat{\boldsymbol{x}}$ is found by minimizing the function $f(\boldsymbol{x})$ [or equivalently maximizing the $\log$ likelihood function $l(\boldsymbol{x})$ ] subject to the constraint $g(\boldsymbol{x})=0$. The gradients and curvatures of the functions $f$ and $g$ computed at $\hat{\boldsymbol{x}}$ then lead to an estimate of the failure probability via equations (28) to (30). Next this result is specialized to obtain the FORM method as one special case.

The random variables that form the vector $x$ in the above analysis are taken as Gaussian. Here Gaussian variables have been chosen as a default in the absence of any other information; the central limit theorem implies that variables tend to be Gaussian unless there is a good reason for them not to be. In case they are not Gaussian it is always possible to transform them to a set of Gaussian uncorrelated random variables using, for example, the transformation of reference [12].

In the applications considered here, $g(\boldsymbol{x})$ can be taken to be the negative of the least stable pole and the 'exact' probability of failure is given by the integral of the joint probability density function of $X$ over the failure region. In general, the resulting integral can be a very difficult quantity to compute since $X$ can have 'very high' dimensions and $g(\boldsymbol{x})$ can have a complex geometry. The FORM approach sets out to obtain an approximate probability of failure by transforming the set of variables in $\boldsymbol{x}$ to a set of uncorrelated Gaussian variables $Z$, each member of which has zero mean and unit standard deviation. The probability of failure is then estimated using

$$
P_{\mathrm{F}}=\Phi(-\beta)
$$

where

$$
\beta^{2}=\min \left\{Z^{\mathrm{T}} Z: g(\boldsymbol{x})=0\right\}
$$

and $\Phi$ is the cumulative normal distribution function. Geometrically, $\beta$ is the shortest distance between $g(Z)$ and the origin. Equation (32) is exact if the safety 
margin $g(Z)$ is a linear function; otherwise the result is an approximation which is based on linearizing the safety margin about the point of closest approach to the origin. Equation (33) is a constrained optimization problem which can be solved numerically provided $g(\boldsymbol{x})$ can be evaluated for a specified value of $\boldsymbol{x}$; the LRR modelling approach used here provides an efficient way of completing this task.

As an illustrative example, consider again the case for which Fig. 7 is a candidate design. Suppose now that the dimensions of the panel are uncertain and taken to be Gaussian random variables with standard deviations of $0.33,0.33$ and $0.066 \mathrm{~mm}$ for length, breadth and thickness respectively. The damping is also assumed to be random with a standard deviation of 3 per cent of the nominal value (all other parameters and the linear quadratic optimal control based controller used can be found in reference [3]). Note also that the relatively large variation in the panel dimensions is intended to make allowance for uncertainties in both the boundary conditions and the actual dimensions themselves.

In a representative result from the Monte Carlo simulations, an ensemble of 1700 plants gave 20 that were unstable and hence a probability of failure of 0.0018 . The open- and closed-loop poles obtained from a sample of 550 of ensemble are shown in Fig. 8, which shows that the poles of the closed-loop system have a very high degree of variability. For example, pole 18 becomes unstable for some of the perturbed plants.

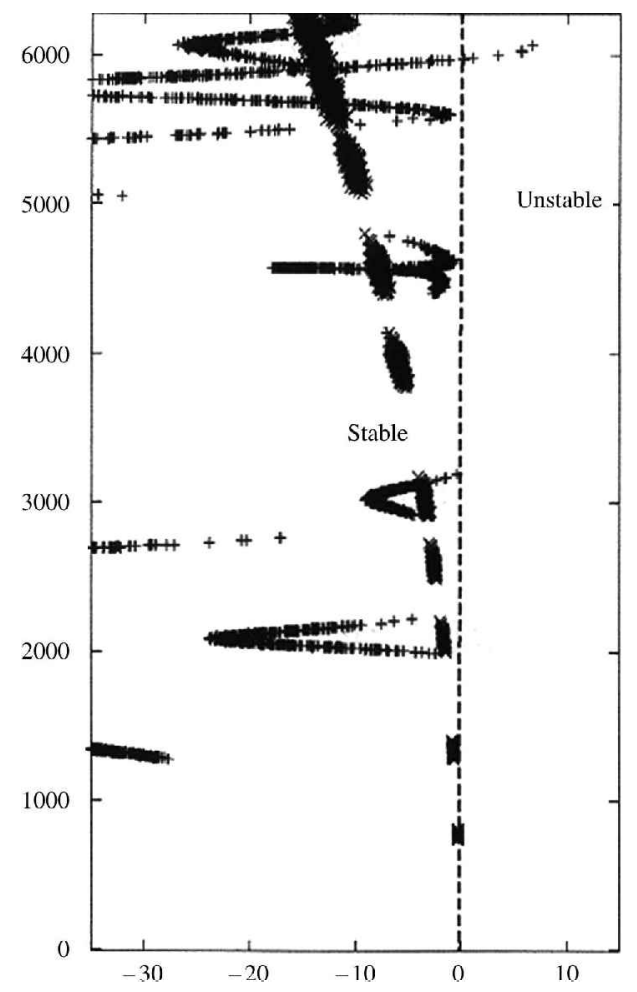

Fig. 8 Poles of the plant $(\times$, open loop; + , closed loop)
The FORM analysis in this case yielded $\beta=2.27$, which corresponds to a probability failure of approximately 0.012. Also, the failure point, i.e. the point satisfying equation (33), lies on the portion of $g(x)$ that is associated with pole 18 and hence both the probability of a failure and the mode of failure are in good agreement with the Monte Carlo results. This analysis also required a small fraction of the computer time needed for the Monte Carlo simulations [typically 30 to 60 calls to the function $g(\boldsymbol{x})]$. In this case, the run-time is reduced by a factor of 30 in comparison to using the Monte Carlo approach alone. Hence the combination of the FORM and the LRR modelling technique is a very efficient method of studying the robustness properties of the actively controlled system.

\section{CONCLUSIONS}

The requirement to suppress microvibrations usually entails the use of an active control scheme. This, in turn, requires a suitable mathematical model on which to base controller design and initial performance predictions prior to experimental implementation for those control laws for which the simulation-based predictions are satisfactory. In this paper, one such mathematical modelling technique developed in previous work has first been summarized. A particular advantage of this method is that it directly permits control system design by any of the currently available linear analysis based methods; here, for illustrative purposes, linear quadratic optimal control has been used.

Given that any model is never a perfect model of the system dynamics, it is essential that the actual quality of the model predictions against measured data is available (if the quality is judged to be 'poor' then clearly predicted behaviour under control action is extremely unlikely to be close enough to the specifications to enable the design exercise to proceed and a 'better' model must be sought). The first significant contribution of this paper is the design and construction of an experimental rig for this model validation task in the form of a mass-loaded panel. The second novel contribution lies in showing that this set-up (mathematical modelling plus the experimental rig) enables probablistic-based studies of the effects of uncertainties in the panel dimensions/materials to be undertaken with little extra preparatory work.

\section{REFERENCES}

1 Stark, H. R. and Stavrinidis, C. ESA microgravity and microdynamics activities-an overview. Acta A stronautica, 1994, 34, 205-221.

2 Zienkiewicz, O. C. The Finite Element Method, 1977 (McGraw-Hill, London). 
3 Aglietti, G. S., Gabriel, S. B., Langley, R. S. and Rogers, E. A modeling technique for active control design studies with application to spacecraft microvibration. J. Acoust. Soc. Am., 1997, 102(4), 2158-2166.

4 Aglietti, G. S. Active control of microvibrations for equipment loaded spacecraft panels. $\mathrm{PhD}$ thesis, University of Southampton, 1999.

5 Aglietti, G. S. and Cunningham, P. R. Is a simple support really that simple? J. Sound Vibr., 2002, 257(2), 321-335.

6 Brennan, M. J., Day, M. J., Elliott, S. J. and Pinnington, R. J. Piezoelectric actuators and sensors. In Proceedings of IUTAM Symposium on Active Control of Vibrations, 1994.

7 Madsen, H. O., Krenk, S. and Lind, N. C. Methods of Structural Safety, 1986 (Prentice-Hall, Englewood Cliffs, New Jersey).
8 Thacker, B. and Millwater, H. NESSUS: a new tool for safer structures. In Technology Today, 1991 (The Southwest Research Institute).

9 Langley, R. S. A unified approach to the probabilistic and possibilistic analysis of uncertain systems. ASCE J. Engng Mechanics, 2000, 126(11), 1163-1172.

10 Rackwitz, R. and Fiessler, B. Structural reliability under combined random load sequences. Computers and Structs, 1978, 9(5), 489-494.

11 Paloheimo, E. and Hannus, M. Structural design based on weighted fractiles. ASCE J. Structural Engng, 1974, 100(St 7), 1367-1378.

12 Rosenblatt, M. Remarks on a multivariable transformation. Ann. Math. Statistics, 1952, 23, 470-472. 